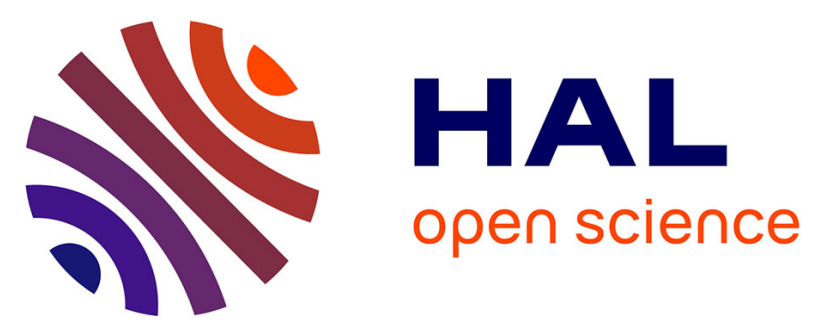

\title{
Fucosylated ubiquitin and orthogonally glycosylated mutant A28C: conceptually new ligands for Burkholderia ambifaria lectin (BambL)
}

Sakonwan Kuhaudomlarp, Linda Cerofolini, Sabrina Santarsia, Emilie Gillon, Silvia Fallarini, Grazia Lombardi, Maxime Denis, Stefano Giuntini, Carolina Valori, Marco Fragai, et al.

\section{To cite this version:}

Sakonwan Kuhaudomlarp, Linda Cerofolini, Sabrina Santarsia, Emilie Gillon, Silvia Fallarini, et al. Fucosylated ubiquitin and orthogonally glycosylated mutant A28C: conceptually new ligands for Burkholderia ambifaria lectin (BambL). Chemical Science, 2020, 10.1039/d0sc03741a . hal-02995036

\section{HAL Id: hal-02995036 \\ https://hal.science/hal-02995036}

Submitted on 8 Nov 2020

HAL is a multi-disciplinary open access archive for the deposit and dissemination of scientific research documents, whether they are published or not. The documents may come from teaching and research institutions in France or abroad, or from public or private research centers.
L'archive ouverte pluridisciplinaire $\mathbf{H A L}$, est destinée au dépôt et à la diffusion de documents scientifiques de niveau recherche, publiés ou non, émanant des établissements d'enseignement et de recherche français ou étrangers, des laboratoires publics ou privés. 


\section{Chemical Science}

Check for updates

Cite this: DOI: 10.1039/d0sc03741a

๑ All publication charges for this article have been paid for by the Royal Society of Chemistry

Received 7th July 2020

Accepted 9th October 2020

DOI: $10.1039 / \mathrm{d} 0$ sc03741a

rsc.li/chemical-science

\section{Fucosylated ubiquitin and orthogonally glycosylated mutant A28C: conceptually new ligands for Burkholderia ambifaria lectin (BambL) $\uparrow$}

\begin{abstract}
Sakonwan Kuhaudomlarp, $\ddagger^{\mathrm{a}}$ Linda Cerofolini, (D) $\dot{\dagger}^{\mathrm{b}}$ Sabrina Santarsia, ${ }^{\mathrm{c}}$ Emilie Gillon, ${ }^{\mathrm{a}}$ Silvia Fallarini, ${ }^{\mathrm{d}}$ Grazia Lombardi, ${ }^{\mathrm{d}}$ Maxime Denis, ${ }^{\mathrm{ce}}$ Stefano Giuntini, ${ }^{\mathrm{cf}}$ Carolina Valori, ${ }^{\mathrm{c}}$ Marco Fragai, ${ }^{* c f}$ Anne Imberty, (D) *a Alessandro Dondoni (D) ${ }^{9}$ and Cristina Nativi (D) *c

Two orthogonal, metal free click reactions, enabled to glycosylate ubiquitin and its mutant A28C forming two protein scaffolds with high affinity for BambL, a lectin from the human pathogen Burkholderia ambifaria. A new fucoside analogue, with high affinity with BambL, firstly synthetized and co-crystallized with the protein target, provided the insights for sugar determinants grafting onto ubiquitin. Three ubiquitin-based glycosides were thus assembled. Fuc-Ub, presented several copies of the fucoside analogue, with proper geometry for multivalent effect; Rha-A28C, displayed one thio-rhamnose, known for its ability to tuning the immunological response; finally, Fuc-Rha-A28C, included both multiple fucoside analogs and the rhamnose residue. Fuc-Ub and Fuc-Rha-A28C ligands proved high affinity for BambL and unprecedented immune modulatory properties towards macrophages activation.
\end{abstract}

\section{Introduction}

Carbohydrate-functionalized proteins are appealing substrates for the development of chemically modified biotherapeutics and proteins-based materials. Indeed, the carbohydrate residues are not just decorative elements as they profoundly affect protein folding, immunogenicity, and stability toward proteases besides controlling biological properties and activities. ${ }^{1}$ In the last decade, it has become clear that proteins can play a role as scaffolds, that is as platforms to localize signalling molecules to a specific part of a cell or to enhance the efficacy of cells crosstalk by co-localizing signalling molecules. ${ }^{2}$ Although the function of scaffold proteins is still matter of investigation, stable and structurally determined proteins assumed a compelling interest

${ }^{a}$ Université Grenoble Alpes, CNRS, CERMAV, 38000 Grenoble, France ${ }^{b}$ CIRMMP, University of Florence, via Sacconi, 650019 Sesto F.no, FI, Italy

${ }^{c}$ Università di Firenze, Department of Chemistry, via della Lastruccia, 3, 13, 50019 Sesto F.no, FI, Italy.E-mail: cristina.nativi@unifi.it

${ }^{d}$ Università del Piemonte Orientale, Department of Pharmaceutical Sciences, 28100 Novara, Italy

${ }^{e}$ Giotto Biotech, via Madonna del Piano, 6, 50019 Sesto F.no, FI, Italy

${ }^{f}$ CERM, via Sacconi, 6, 50019 Sesto F.no, FI, Italy

${ }^{g}$ Interdisciplinary Center for the Study of Inflammation, University of Ferrara, 44121 Ferrara, Italy

$\dagger$ Electronic supplementary information (ESI) available: Synthesis, characterization data, all experimental details, crystallographic data, and supplementary figures, tables and spectra. PDB ID 6ZFC (Deposition ID D_1292107260) and the associated experimental data have been deposited with release instructions 'HPUB'. See DOI: 10.1039/d0sc03741a

\$ These authors contributed equally. as bio-compatible platforms to display clusters of glycosidic ligands. ${ }^{3}$

Ubiquitin (Ub) is a fully characterized, widespread protein bearing seven lysine residues in its wild-type form (WT-Ub). Ubiquitination is a reversible modification in which the terminal glycine residue of $\mathrm{Ub}$ is attached to a substrate protein. This physiological modification is essential for a great number of biological events, including the regulation of innate and adaptive immune responses. ${ }^{4}$ Ub features eight $\mathrm{NH}_{2}$-groups (including seven residues of Lys and the N-terminus), five of which are exposed to the solvent on the same side of the protein surface with a random distribution. ${ }^{5}$ Ub thus combines the requisites of a scaffold protein and herein we investigate its possible role for presenting glycan ligands to bacterial receptors and triggering feedback signals. ${ }^{2}$

Lectins are glycan-binding proteins playing pivotal roles in cell-cell recognition, signaling and infection. ${ }^{6}$ A plethora of virus, bacteria and fungi take advantage of lectins to adhere and infect host cells by binding glycoconjugates exposed on cell surface. Although surface glycans generally have complex structures, lectins commonly recognize terminal mono- and disaccharides. The recognition of small epitopes and the carbohydrate-lectin binding mode, can indeed account for the binding of lectins with diverse biological functions to the same ligand. ${ }^{7}$ Well-known examples are bacterial soluble lectins LecB of Pseudomonas aeruginos $a^{8}$ and BambL of Burkholderia ambifaria. ${ }^{9}$ LecB and BambL are fucose-binding lectins, characterized by a high affinity (low micromolar range) for fucose. Both have the ability to bind to fucosylated oligosaccharides present on human tissues and are involved in P. aeruginosa and $B$. 
ambifaria human infections, with serious consequences when they affect immune-compromised or cystitis fibrosis patients. LecB and BambL are therefore interesting targets to block bacterial adhesion..$^{\mathbf{1 0 , 1 1}}$

The development of epitope analogues to hamper pathogens adhesion to host cells and contrast the resulting infection without inducing antimicrobic resistance is currently a wellestablished strategy. In this context, attractive fucose analogues targeting LecB have been reported. ${ }^{12}$ As for BambL, we recently proposed the aryl- $\alpha$-O-fucosyl analogue 1 (Fig. 1), characterized by an unprecedented specificity for the BambL binding site. ${ }^{13}$ Indeed, 1 does not bind to other fucose-binding lectins like bacterial LecB or human DC-SIGN (Dendritic CellSpecific Intercellular adhesion molecule-3-Grabbing Nonintegrin). It is worthy of note that $\mathbf{1}$ displays a high affinity for AFL, the lectin from airborne fungal pathogen Aspergillus fumigatus, which has strong structural similarities to BambL. ${ }^{\mathbf{1 4}}$ These two lectins from lung pathogens are constituted of a repeat of similar small $\beta$-sheets of approximately 40 amino acids and can bind up to six fucose residues (Fig. 1). ${ }^{15}$

Lectins commonly contain several binding sites which are formed by oligomerization or sequence repetition. Clustered glycosyl ligands targeting multiple lectin binding sites greatly increase ligands binding affinity through avidity. ${ }^{7}$ For example, fucosylated glyco-clusters can bind efficiently to $\operatorname{LecB}^{\mathbf{1 6 , 1 7}}$ or BambL. ${ }^{18,19}$ with nanomolar affinity. Bridging the concepts of physiologically stable glycosyl analogues and multivalency, we synthesized the pre-organized fucosyl cyclohexapeptide 2 (Fig. 1) for the presentation of six residues of the fucosyl analogue $\mathbf{1 .}^{\mathbf{1 4}}$ The resulting hexavalent construct (2), showed a high-avidity binding $v s$. BambL $\left(K_{\mathrm{d}}=14 \mathrm{nM}\right)$ and $\operatorname{AFL}\left(K_{\mathrm{d}}=44\right.$ $\mathrm{nM})$. Nonetheless, molecular modeling performed to investigate the binding mode of 2 with BambL suggested that the high binding affinity assessed was likely due to the cross-linking of sites on discrete lectins rather than to the binding of adjacent sites in a single trimeric lectin.

Herein, taking advantage from the selective ligand $\mathbf{1}$ and proposing Ub as protein scaffold, we report on conceptually new ligands with high affinity for BambL and addressing adjacent sites in a single lectin. An analog of $\mathbf{1}$ was synthetized, assayed for binding to BambL and AFL and the structure of its complex with BambL was determined. Fucosylated Ub was then prepared and assayed for its affinity for BambL. In addition to fucosylation, orthogonal glycosylation of the engineered Ub mutant
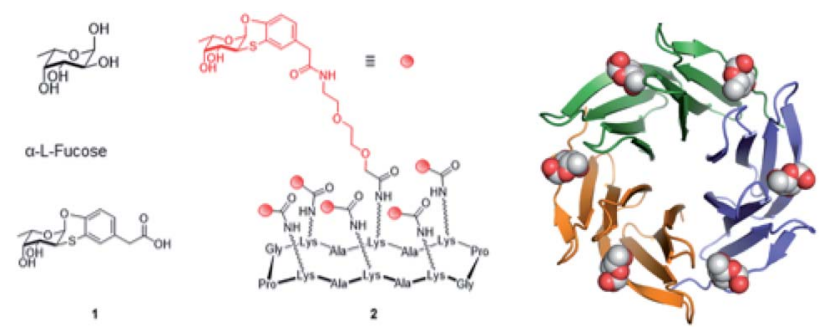

Fig. 1 Structure of L-fucose, fucosyl analogue 1, hexavalent fucosyl cyclopeptide 2 and BambL lectin, as trimer with six fucose residues in binding sites (adapted from crystal structure with PDB code 3ZZV).
A28C with a rhamnose residue, was also considered. In fact, rhamnose-containing plant polysaccharides have recently been demonstrated to modulate activities in macrophages in in vitro inflammatory models. ${ }^{20,21}$ The three ligands, namely Fuc-Ub, Rha-A28C and Fuc-Rha-A28C, in vitro biologically characterized, have been tested for their ability to modulate macrophage activation either in resting conditions or after lipopolysaccharide (LPS) stimuli.

\section{Results and discussion}

\section{Synthesis of the hydroxypropyl derivative 3}

To design the new Ub-based ligands, we first aimed at better characterizing the binding mode of the $\alpha$-O-aryl fucoside 1 to BambL by synthesizing the fucosyl derivative 3 (Scheme 1), which is similar to $\mathbf{1}$ but with an additional five-atom tag linked, through an amidic bond, to the $\alpha$-aryl fucoside. Compound 3 was obtained in three steps from methyl ester $4^{\mathbf{1 3}^{3}}$ Ester 4 was firstly hydrolyzed with $\mathrm{LiOH}$ in $\mathrm{THF}-\mathrm{H}_{2} \mathrm{O}(2 \mathrm{~h}$, room temperature) to form the free carboxylate 5 (85\%); under these conditions, the partial deprotection of the ditert-butylsilylidene group took place (see ESI $\dagger$ for details). Carboxylate 5 was then activated in situ with 2-(1H-benzotriazol-1-yl)-1,1,3,3tetramethyluronium hexafluorophosphate (HBTU) and $\mathrm{N}$ methyl morpholine (NMM) and reacted at room temperature with 3-amino-1-propanol in DMF as solvent, to afford 6 (50\%). Final deprotection of silyl derivative 6 with freshly prepared TBAHF ( $2 \mathrm{M}$, THF, $40{ }^{\circ} \mathrm{C}$, overnight) enable the isolation of fucoside 3 as white solid (quant.) (Scheme 1).

\section{Binding of derivative 3 to BambL and AFL}

At first, the binding of 3 was assessed by ITC (Isothermal Titration Calorimetry) towards the two lectins from pathogens, BambL and AFL and compared to $\alpha \mathbf{M e F u c}$ as control. Dissociation constants and thermodynamic data are listed in Table 1.

Compound 3 binds efficiently to BambL, but the curve could not be fitted with a 1:1 binding model (Fig. 2). As observed before with compound $1,{ }^{13}$ a two-site model has to be used, resulting in the determination of two binding modes, the first one with high affinity $\left(K_{\mathrm{d}}=240 \mathrm{nM}\right)$ and strong enthalpy contribution $\left(\Delta H=-35 \mathrm{~kJ} \mathrm{~mol}^{-1}\right)$ and the second one with weaker binding $\left(K_{\mathrm{d}}=23 \mu \mathrm{M}, \Delta H=-22 \mathrm{~kJ} \mathrm{~mol}^{-1}\right)$. In both cases, a small favorable entropy term participates to the binding

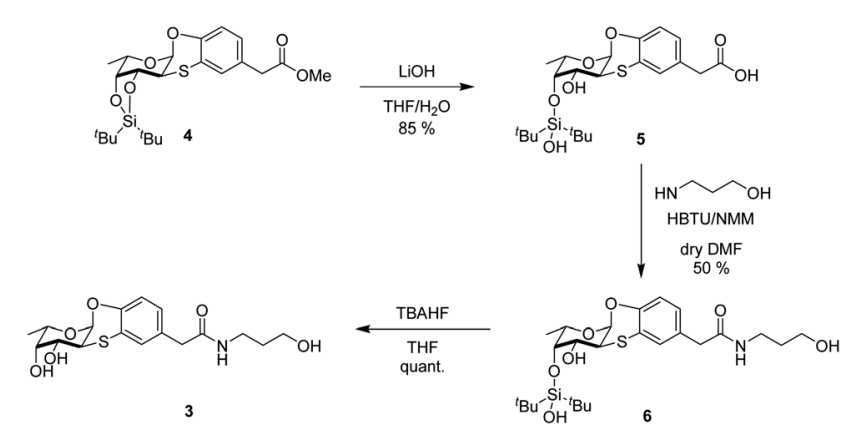

Scheme 1 Synthesis of fucosyl derivative 3. 
Table 1 Microcalorimetry data for monovalent compound 3 interacting with BambL and AFL compared to $\alpha$ MeFuc as control. Standard deviations over two experiments are lower than $20 \%$. BambL is a trimer with 2 blades per monomer, while AFL is a monomer with 6 blades

\begin{tabular}{llllll}
\hline & Lectin & $n$ & $K_{\mathrm{d}}(\mu \mathrm{M})$ & $\Delta H\left(\mathrm{~kJ} \mathrm{~mol}^{-1}\right)$ & $-T \Delta S\left(\mathrm{~kJ} \mathrm{~mol}^{-1}\right)$ \\
\hline 3 & BambL & 2 sites $^{a}$ & $0.24 / 23$ & $-35.0 /-21.8$ & $-3.0 /-4.7$ \\
& AFL & 3.9 & 30.3 & -35.7 & 8.8 \\
aMeFuc & BambL & 1.9 & 1.02 & -45.5 & 11.4 \\
& AFL & 3.4 & 76.4 & -45.2 & 21.8 \\
\multicolumn{5}{c}{ Two independent sites with $n=1}$. &
\end{tabular}

(A)

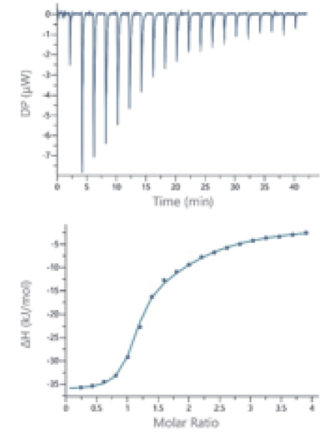

(B)

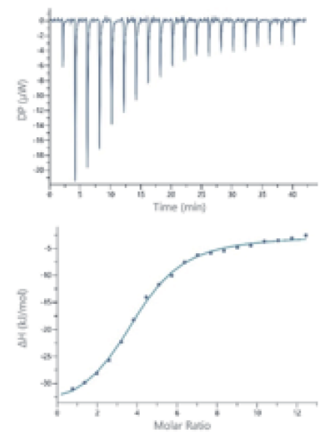

Fig. 2 (A) Thermograms (top) and titration curves (bottom) obtained from the titration of (A) BambL $(50 \mu \mathrm{M})$ and (B) AFL $(50 \mu \mathrm{M})$ by compound 3 (both $3 \mathrm{mM}$ ). Fitting procedure was performed using a two-site model for BambL and with a one site model for AFL.

energy. The significant change in entropy contribution for the binding of compound 3 compared to $\alpha$ MeFuc confirms previous ITC data obtained with compound $\mathbf{1 .}^{13}$ Binding of the aryl analogues can be described as both enthalpy and entropy driven, probably due to the increase in rigidity and hydrophobicity from the additional ring system. The two binding sites of BambL were previously reported to have similar affinity towards fucose, but different behavior towards the $\mathrm{H}$ type 2 blood group trisaccharide ${ }^{9}$ and towards compound $1 .^{13}$ The control experiments with $\alpha$ MeFuc are in agreement with those previously reported, $, 913,15,22$ and this validates the presence of two equivalent fucose binding sites with an affinity for fucose of approx. 1 $\mu \mathrm{M}$ in each BambL monomer. The stoichiometry of binding to AFL is lower than the six binding sites per propeller, but the occurrence of differences in affinity was reported before. ${ }^{15}$

\section{Crystal structure of BambL complexed with derivative 3}

We then attempted to co-crystallize BambL with $\mathbf{1}$ and 3. Only co-crystallization trials with 3 lead to the formation of crystals suitable for diffraction. The diffraction data were collected on beamline PX1 at the Soleil synchrotron to $1.65 \AA$ resolution in the $I 121$ space group. The structure was solved by molecular replacement using $3 \mathrm{ZW} 0$ as a template (detailed statistics for data collection and processing are shown in Table S1†) (Fig. 3).

The asymmetric unit contains six monomers of BambL assembled into two distinct 6-bladed $\beta$-propellers (chain ABC
(A)
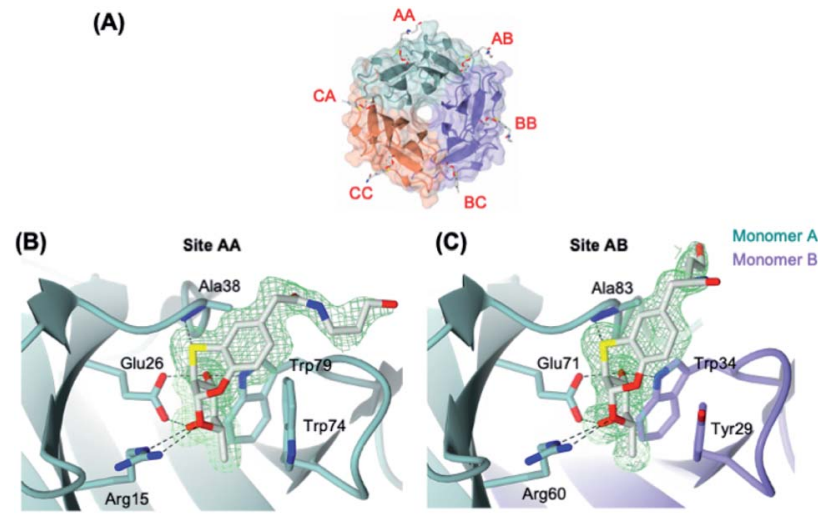

(D)

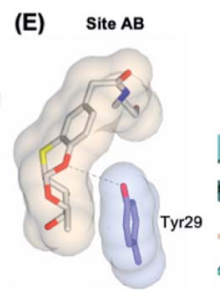

(F) Site AA
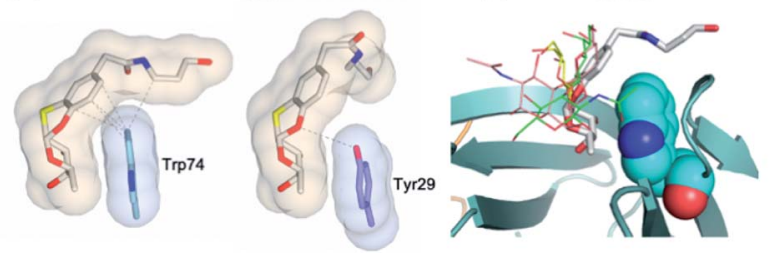

Fig. 3 (A) Protein crystal of BambL co-crystallised with 3. The binding sites are named after the amino acid chains that constitute the binding sites. (B) and (C) The binding sites of ligand 3 in BambL intra and inter sites. Green mesh represents the $2 \mathrm{mFo}$-DFc electron density maps for the ligand contoured at $1 \sigma$. (D) and (E) Surface and contact analysis of 3 with intra (site $A A$ ) and inter binding sites (site $A B$ ). The surfaces are shown in blobs and contacts within $4 \AA$ are shown as grey dashes. Surface and contact analysis of all sites can be found in Fig. S2. $\dagger$ (F) Superimposition of fucosylated ligands in intramolecular site of different crystalline complex, with 3 represented as thick lines, $\mathrm{H}$-type 1 (green, 3ZW2), H-type 2 (pink, 3ZZV), blood group B (yellow 3ZWE).

and chain DEF) that are related by crystallographic symmetry operator. Due to the pseudo-crystallography symmetry relation between the two $\beta$-propellers, we only describe herein chain $\mathrm{ABC}$ in full detail. Six molecules of 3 were present per $\beta$ propeller ( 2 molecules per monomer) and occupied the same binding pockets as those described for native fucosylated glycans (Fig. 3A). The electron density maps clearly show the aromatic aglycone moiety of the ligand in the same configuration in all of the binding sites (Fig. 3B and C). In contrast to the fucosyl and aromatic aglycone moieties, the electron density for the aliphatic portion of 3 , which is flexible and exposed to the solvent, was found intact only in site $\mathrm{AA}$ and $\mathrm{AB}$ (Fig. 3B and C). The flexibility of the aliphatic portion and the lack of its interaction with the protein explain the absence of its electron density in the other binding sites. However, the fucose and aryl groups are clearly visible in all sites (Fig. S1†). The fucosemimicking portion of the ligand 3 interacts extensively via hydrogen bonds with the protein in the same manner as described for the native fucose. ${ }^{9}$ In contrast, the interaction through hydrogen bonds between the aglycone part and the protein is only limited to a hydrogen bond between $\mathrm{S}$ atom and the $\mathrm{NH}$ group of Ala38, which is in agreement with a reported observation from a molecular modelling of BambL with $\mathbf{1 .}^{13}$

BambL displays two types of fucose-binding sites: the sites located within a monomer (intra or Type I) and the sites located 
between two adjacent monomers (inter or Type II), which are distinguished by the presence of Trp74 (Type I) or Tyr29 (Type II) (Fig. 3B and C). We hypothesized that the slight structural difference in the two binding sites may account for the two distinct binding affinities of BambL to compound 3 that were observed in ITC experiment. Analysis of interface and van der Waals's contacts between compound 3 and the aromatic residues (Trp74 or Tyr29) showed that Tyr29 forms contacts only with the fucosyl moiety, whilst Trp74 forms more extensive contacts with the fucosyl and the aglycone moiety, in particular to the 6C ring (Fig. 3D, E and S2 $\dagger$ ). Such stacking between aromatic residues and the face of pyranose ring has well been described in complexes between proteins and carbohydrates, involving $\mathrm{CH} / \pi$ bonds. ${ }^{23}$ The extensive contacts observed between the aryl group of the ligand and the Trp residue in Type I binding sites clearly explain the stronger binding affinity $\left(K_{\mathrm{d}}=\right.$ $0.24 \mu \mathrm{M})$ as opposed to the 100 -fold weaker binding in Type II site $\left(K_{\mathrm{d}}=23 \mu \mathrm{M}\right)$. These distinct affinities between the two sites have also been observed in the interaction of BambL with $\mathrm{H}$ type II oligosaccharide. ${ }^{9}$ When compared to previously characterized oligosaccharidic ligands (H-type I, H-type II and blood group B) ${ }^{9}$ (Fig. $3 \mathrm{~F}$ ), compound 3 clearly results in closer contact to the Trp74, in Type I site, which could explain the much higher affinity at this site in comparison to the affinities for $\mathrm{H}$ type I $\left(K_{\mathrm{d}}=26.1 \pm 0.1 \mu \mathrm{M}\right)$ and blood group $\mathrm{B}\left(K_{\mathrm{d}}=95.3 \pm 1.8\right.$ $\mu \mathrm{M})$. Interestingly, $\mathrm{H}$-type II, which also exhibits two-site binding behavior $\left(K_{\mathrm{d}}=0.56 \pm 0.01\right.$ and $\left.14 \pm 0.3 \mu \mathrm{M}\right)$, established a water-mediated hydrogen bond between 06 of GlcNAc and nitrogen of Tryp-74, which demonstrates an alternative binding strategy that enhances the affinity towards BambL Type I site.

\section{Synthesis of fucosyl derivatives 7 and ubiquitin glycosylation}

Crystal structure of BambL complexed with 3 clearly showed that the chain linked to the carboxylic residue of the ligand does not participate nor affect the binding with the lectin. Moreover, since the chain terminal hydroxyl protrudes outside the lectin, it represents a convenient hook to insert a spacer to bridge fucoside $\mathbf{1}$ and a suitable multivalent scaffold to form a glycocluster to address the multiple sites of the pathogen lectin.

Glycosylation $^{24,25}$ is an important modification of natural proteins because the resulting material, having a precise structure and composition may show new chemistry and stability. The introduction of carbohydrate moieties in proteins is by far not a trivial operation as it must be carried out by efficient and chemoselective ligation reactions that occur under mild conditions compatible with protein chemical stability. The rich arsenal of click reactions include metal-free processes that are suitable for this endeavour. To this aim we selected the addition of $N$-hydroxysuccinimide (NHS) ester to amines. Ub is a small $8.6 \mathrm{kDa}$ protein bearing seven Lys sidechains, in its wildtype form (WT-Ub). We therefore planned to take advantage from the seven Lys $\varepsilon-\mathrm{NH}_{2}$ residues and from the $\mathrm{N}$-terminus to link up to eight residues of fucoside 7 (Scheme 2) through amide-bond formation. ${ }^{26}$ With respect to fucosyl derivative 7 features a longer aliphatic chain, to properly exhibit the sugar

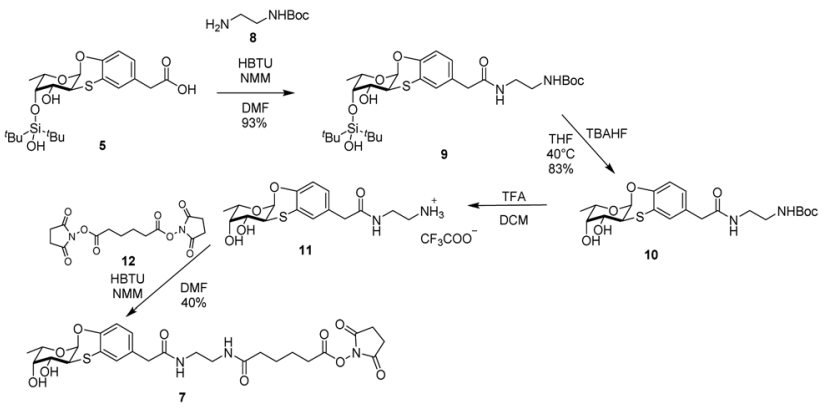

Scheme 2 Synthesis of fucosyl derivative 7.

moiety on protein surface, ${ }^{3}$ ending with a NHS activated carboxylic group. Compound 7 was synthesized in four steps from carboxylic acid 5 (see Scheme 1) by treatment in DMF with HBTU and NMM, and reaction with mono-Boc protected ethylenediamine 8, to form the NHBoc derivative 9 (93\%). Compound 9 was deprotected by removal of silyl ether group (TBHF, THF, 10, 83\%) and Boc residue (TFA, DCM) to afford 11 as trifluoroacetic salt. Fucosyl derivative $\mathbf{1 1}$ was finally reacted with NHS-adipoyl derivative 12 (HBTU, NMM, DMF, rt) ${ }^{27}$ to yield, after purification, the activated ester 7 as white solid $(40 \%$ over three steps) (Scheme 2).

The ester 7 was thus used to decorate WT-Ub. As described, $\S$ a solution of highly concentrated recombinant WT-Ub $(450 \mu \mathrm{M}$ in phosphate buffer at $\mathrm{pH} 7.5)$ was supplied with a large excess of 7 (70 eq., i.e. 10 eq. for each $\varepsilon-\mathrm{NH}_{2}$ group of lysine residues) to promote the largest degree of conjugation. The reaction was carried out at low temperature $\left(4^{\circ} \mathrm{C}\right)$ to reduce competitive NHS-ester hydrolysis (see ESI $\dagger$ ). The evolution of conjugation reaction was monitored by gel electrophoresis, then, when all free WT-Ub was functionalized (100\% yield of conjugation), the final Fuc-Ub conjugate was recovered and washed with fresh buffer to remove unreacted 7. Fuc-Ub characterization by MALDI-MS, SPR and NMR techniques showed that up to eight fucoside residues were linked to $\mathrm{Ub}$ (on the average of 5.6 graphed fucosides, see ESI Fig. S3†).

\section{Binding assessment of Fuc-Ub to BambL by SPR}

Assays to measure the binding of fucosylated ubiquitin (Fuc-Ub) to BambL were initially performed by ITC; however, no clear thermogram could be obtained, indicating that the binding was either not sufficiently endothermic or exothermic for this method, or that the affinity was low. SPR was then considered with BambL immobilized on the chips. The integrity of the lectin was first checked with injection of fucosylated tetrasaccharides (Sialyl Lewis X) that demonstrated the expected binding. Injection of Fuc-Ub with concentration varying from $1 \mathrm{nM}$ to $1 \mu \mathrm{M}$ resulted in strong binding events (Fig. 4). The shape of the sensorgrams with slow association, and even slower dissociation phase are typical for multivalent binding (rebinding effect). As expected in such case, it was not possible to fit a classical 1:1 binding model with the curve for determination of $k_{\text {on }}$ and $k_{\text {off. }}$. The affinity was therefore determined by affinity analysis of binding response at steady state (Fig. 4B), 
(A)

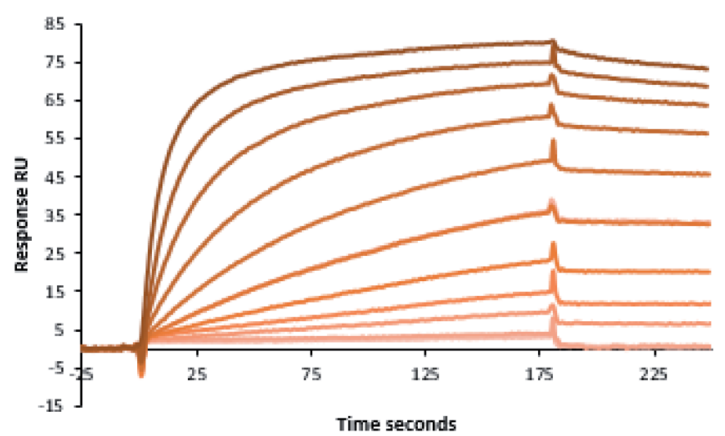

(B)

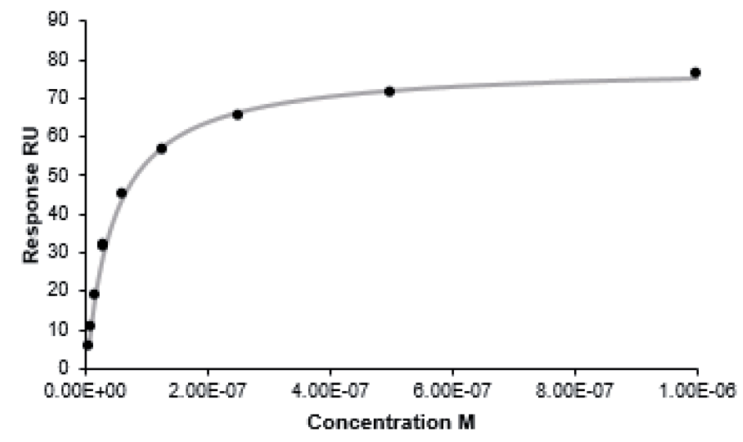

Fig. 4 SPR analysis of interaction between Fuc-Ub and immobilized BambL. (A) Sensorgram of multiple cycle affinity analysis consisting of injections of increasing concentration ( $1 \mathrm{nM}$ to $1 \mu \mathrm{M})$ of Fuc-Ub. (B) Affinity analysis based on data from (A).

and a $K_{\mathrm{d}}$ value of $46.7 \mathrm{nM}$ was obtained. This observed high affinity together with the absence of enthalpy signals observed by ITC indicate that the interaction is entropy driven.

\section{NMR data of WT-Ub conjugated with multiple copies of fucosyl derivative 7 (Fuc-Ub)}

Fucosylated WT-Ub was analyzed by NMR. $2 \mathrm{D}{ }^{1} \mathrm{H}^{1}{ }^{15} \mathrm{~N}$ HSQC spectra were recorded before and after glycosylation to assess the reaction outcome (Fig. 5). After reaction of WT-Ub with 7, eight new signals appeared in the right bottom region of the ${ }^{1} \mathrm{H}^{-15} \mathrm{~N}$ HSQC spectrum. These signals correspond to the amide group ${ }^{26}$ generated by functionalization of the $\varepsilon-\mathrm{NH}_{2}$ group of Lys side-chains and the N-terminus, with fucosyl derivative 7.

The functionalization of the Lys sidechains also accounts for the chemical shift perturbations of $\mathrm{H}^{\mathrm{N}}$ backbone signals of the residues neighboring the functionalized Lys residues. As reported in Fig. 6 the chemical shift perturbation (CSP) is very pronounced for many lysine residues and for their neighboring residues, while for Lys- 48 and Lys-63 the CSP is significant only for the lysine residues themselves. The latter lysine residues are, indeed, located on more mobile regions of the protein structure.

The interaction of Fuc-Ub with BambL was also investigated by NMR, acquiring $2 \mathrm{D}^{1} \mathrm{H}^{-15} \mathrm{~N}$ HSQC spectra of ${ }^{15} \mathrm{~N}$-isotopically enriched Fuc-Ub before and after the addition of increasing aliquots of a solution of BambL. After the first addition of BambL (at the monomer concentration of $50 \mu \mathrm{M}$, i.e. in a $0.5: 1$ ratio of monomeric BambL : Fuc-Ub) the intensity of the signals

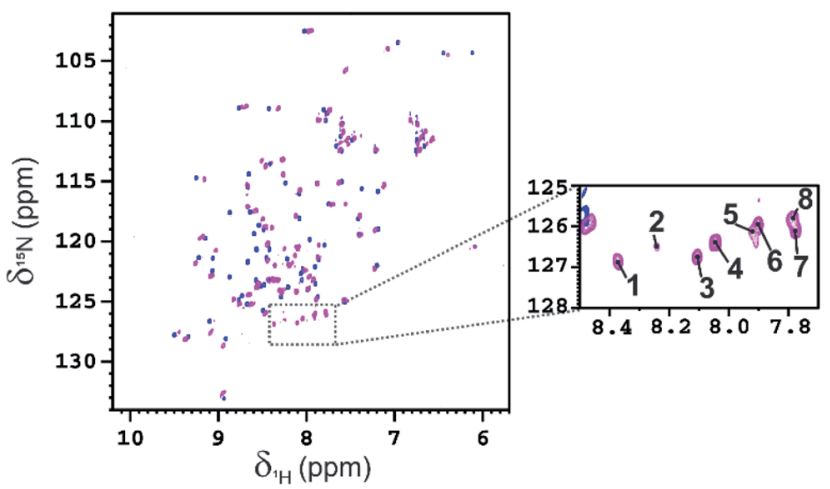

Fig. $52 \mathrm{D}{ }^{1} \mathrm{H}-{ }^{15} \mathrm{~N} \mathrm{HSQC}$ spectra of WT-Ub (blue) and WT-Ub functionalized with the fucosyl derivative 7 on the Lys sidechains (Fuc-Ub, magenta) acquired at $298 \mathrm{~K}$ and $700 \mathrm{MHz}$. The signals in the area, selected by the dashed lines (enlarged on the right), correspond to the new amide groups obtained by the conjugation of the $\varepsilon-\mathrm{NH}_{2}$ group of lysine sidechains and the $\mathrm{N}$-terminus with fucoside 7.

of Fuc-Ub in the $2 \mathrm{D}^{1} \mathrm{H}^{-15} \mathrm{~N}$ HSQC spectrum globally decreased by $\sim 30 \%$ (see Fig. 7). After a second addition of BambL (at the monomer concentration of $100 \mu \mathrm{M}$, i.e. in a $1: 1$ ratio of monomeric BambL : Fuc-Ub), the residual Fuc-Ub NMR signals completely disappeared in the ${ }^{1} \mathrm{H}^{-15} \mathrm{~N}$ HSQC spectrum. The high molecular weight of complexes formed between Fuc-Ub and BambL, increases the transverse relaxation rates with a resulting line broadening which prevents the observation of the signals of Fuc-Ub bound to BambL in the $2 \mathrm{D}^{1} \mathrm{H}^{-15} \mathrm{~N}$ HSQC spectrum.

A SEC-MALS-QELS analysis performed on the NMR sample shows the presence of several species, most of them with an average molar mass of 48-71 kDa (see Fig. S8 $\dagger$ ).

\section{In vitro properties of orthogonally glycosylated Ub mutant A28C}

Synthesis of rhamnosyl derivative 13. Since rhamnosecontaining polysaccharides showed significant modulatory

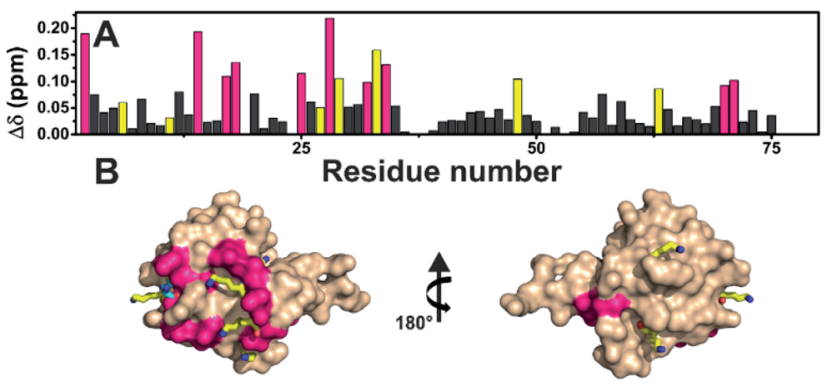

Fig. 6 (A) Graphical representation of the per residue chemical shift perturbation (CSP) of wild type-Ub functionalized on the lysine side chains with fucosyl derivative 7 with respect to the free protein. The CSP has been evaluated according to the formula $\Delta \delta=1 / 2 \sqrt{\left(\Delta \delta_{H}\right)^{2}+\left(\Delta \delta_{N} / 5\right)^{2}}$. The residues exhibiting the highest CSPs have been highlighted in pink, while the lysine residues are in yellow. (B) Surface representation of Ub (PDB: 1UBQ) with highlighted in pink the residues exhibiting the highest CSP; the lysine residues are represented as yellow sticks, and the $\mathrm{N}$-terminus as cyan sticks. 


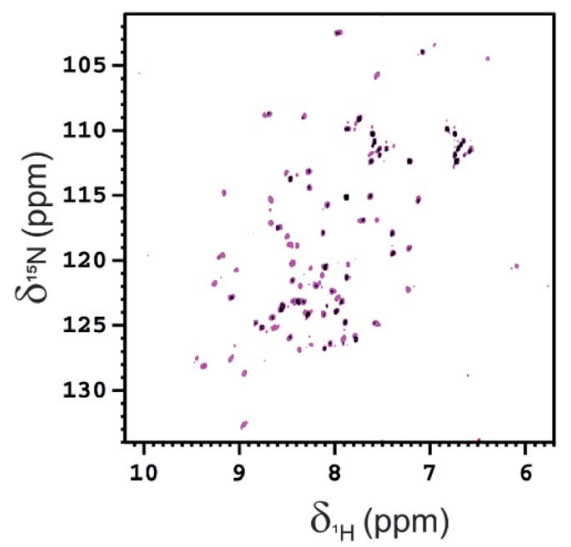

Fig. $72 \mathrm{D}{ }^{1} \mathrm{H}-{ }^{15} \mathrm{~N}$ HSQC spectra of Fuc-Ub $(100 \mu \mathrm{M}$, magenta) and Fuc-Ub in the presence of a sub-stoichiometric amount ( $50 \mu \mathrm{M}$, black) of BambL acquired at $298 \mathrm{~K}$ and $700 \mathrm{MHz}$. The protein was in $20 \mathrm{mM}$ Tris buffer at $\mathrm{pH} 7.5$ with $100 \mathrm{NaCl}$. The signals that have not completely disappeared in the black spectrum belong mainly to residues located on mobile regions (G10, S20, D21, T22, D32, D39, R42, F45, Q49 R54, D58, T66, R72, L73, G76).

activities in macrophages in in vitro inflammatory models, ${ }^{20,21}$ we also introduced this monosaccharide onto Ub to build up a conceptually new BambL inhibitor with immunomodulating properties. We thus biotechnologically expressed the Ub mutant A28C, where alanine 28 was replaced by one cysteine residue (see $\mathrm{ESI}^{\dagger}$ ). This mutant, presenting seven residues of Lys and one residue of Cys, can orthogonally be glycosylated to display different saccharidic moieties. Thus, we functionalized the Cys residue with rhamnose derivative 13, while the Lys residues and the $\mathrm{N}$-terminus were functionalized, as described above, with fucoside 7 (Fig. 8).

The rhamnosyl derivative $\mathbf{1 3}$ was synthesized by reacting the commercially available peracetylated L-rhamnose with allylthiol and catalytic $\mathrm{BF}_{3} \mathrm{OEt}_{2}$ at $0{ }^{\circ} \mathrm{C}$ in dichloromethane as solvent, to afford the thio-glycoside $\mathbf{1 4}(\mathbf{7 0 \% )}$ as single $\alpha$-anomer. The acetyl groups removal under Zemplén conditions ${ }^{28}$ afforded the fully unprotected thio-allyl derivative 13 (54\%) (Scheme 3 and ESI $\dagger$ ).

Orthogonal glycosylation of Ub mutant and NMR data of Fuc-Rha-A28C. The conjugation of Ub-A28C with 13 was efficiently performed by using another classical click reactions namely the photoinduced thiol-ene coupling (TEC) through the

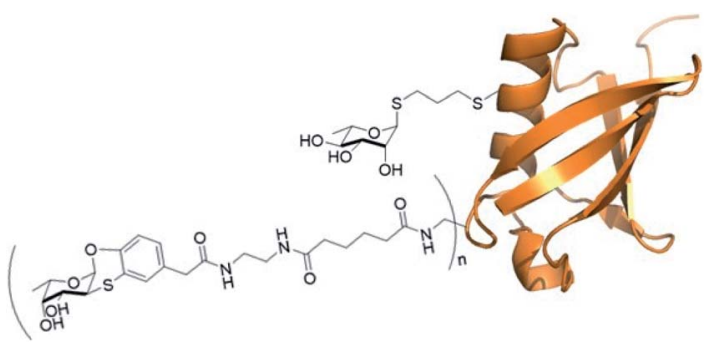

Fig. 8 Ub mutant $\mathrm{A} 28 \mathrm{C}$ decorated with residues of fucosyl derivative 7 $(n=$ on average of 5.6) and one residue of rhamnose derivative 13 (adapted from crystal structure $1 \cup B Q$ ).

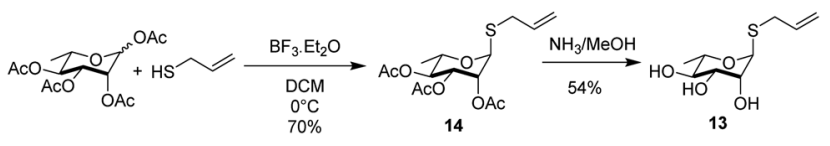

Scheme 3 Synthesis of rhamnosyl derivative 13

formation of a thioether bond. ${ }^{29}$ To a solution of Ub-A28C (450 $\mu \mathrm{M}$ in NaPi $150 \mathrm{mM}, \mathrm{pH} 7.5$ ) were added an excess of 13 and of DPAP as photo-initiator, and the reaction mixture was irradiated at $365 \mathrm{~nm} .{ }^{30}$ The reaction was monitored by $2 \mathrm{D}{ }^{1} \mathrm{H}-{ }^{15} \mathrm{~N}$ HSQC spectra, and reached completion within only 20 minutes. Unreacted 13 and DPAP were washed away and NMR spectra confirmed the formation of Ub-A28C conjugated with a residue of Rha (Rha-A28C) (Fig. S4 $\dagger$ ). The formation of the thioether bond was supported by the shifting of the signals belonging to the residues close to the cysteine (Fig. S5 $\dagger$ ). Rha-A28C was subsequently glycosylated as described above, on the $\varepsilon-\mathrm{NH}_{2}$ group of Lys sidechains and the N-terminus with up to eight residues of fucoside 7. After purification, the orthogonally conjugated protein, Fuc-Rha-A28C, was analyzed by $2 \mathrm{D}^{1} \mathrm{H}-{ }^{15} \mathrm{~N}$ HSQC spectra (Fig. S6†). Eight new signals appeared in the right bottom region of the $2 \mathrm{D}{ }^{1} \mathrm{H}-{ }^{15} \mathrm{~N}$ HSQC spectra, because of the newly formed amide groups after the conjugation of the seven lysine sidechains and the N-terminus with derivative 7 . The chemical shift perturbation of Fuc-Rham-A28C is shown in Fig. S7 (see ESI $\dagger$ ).

NMR is also an appropriate technique for characterizing the interaction between glycoconjugates and protein receptors. ${ }^{31}$ The interaction of functionalized Ub-A28C, Fuc-Rha-A28C, with BambL was also investigated acquiring $2 \mathrm{D}{ }^{1} \mathrm{H}-{ }^{15} \mathrm{~N} \quad \mathrm{HSQC}$ spectra. After the first addition of the lectin (at the monomer concentration of $50 \mu \mathrm{M}$, i.e. in a $0.5: 1$ ratio of monomeric BambL : Fuc-Rha-A28C) the global intensity of the protein signals decreased $\sim 40 \%$ (see Fig. 9). As observed for Fuc-Ub, after a second addition of BambL (at the monomer concentration of $100 \mu \mathrm{M}$, i.e. in a $1: 1$ ratio of monomeric BambL : FucRha-A28C), the residual NMR signals of Fuc-Rha-A28C completely disappeared in the ${ }^{1} \mathrm{H}^{15}{ }^{15}$ HSQC spectrum. The affinity of Fuc-Rha-A28C for BambL appeared, thus, to be in the same range of that assessed for Fuc-Ub.

To rule out a possible interaction of BambL with the Rhap conjugated to A28C and confirm the selectivity of the interaction with fucoside 1, BambL was added to a solution of RhaA28C. After the addition of the same amount of BambL which induced the decreasing of Fuc-Rha-A28C signals, no effect was visible in the $2 \mathrm{D}{ }^{1} \mathrm{H}^{15}{ }^{15} \mathrm{~N}$ HQC spectra of Rha-A28C (Fig. 10). The same was registered by adding an excess of BambL. These data allowed to exclude an interaction between Rhap and the lectin binding sites and confirmed the involvement of only fucoside residues in BambL-Ub binding.

In vitro immunomodulation effects of Fuc-Ub and Fuc-RhaA28C. Macrophages, being the major effector cells of innate immune system, were chosen as a suitable in vitro model to evaluate possible immune modulatory activities of the new synthetized compounds. ${ }^{32}$ To exclude that alteration of macrophage functions was due to intrinsic compound toxicity, firstly, 


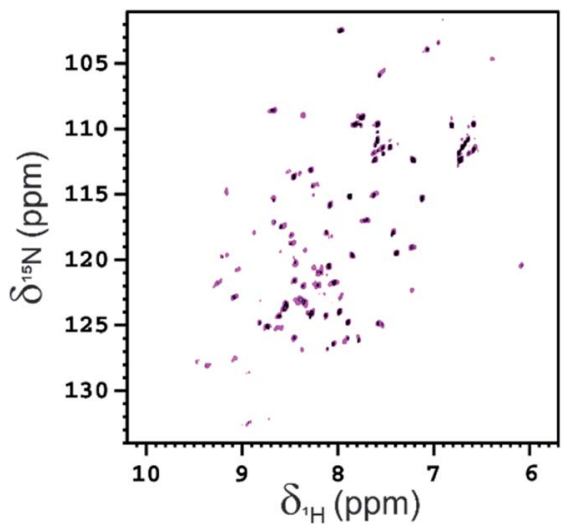

Fig. $92 \mathrm{D}^{1} \mathrm{H}-{ }^{15} \mathrm{~N}$ HSQC spectra of Fuc-Rha-A28C (100 $\mu \mathrm{M}$, magenta) and Fuc-Rha-A28C in the presence of a sub-stoichiometric amount (50 $\mu \mathrm{M}$, black) of BambL, acquired at $298 \mathrm{~K}$ and $700 \mathrm{MHz}$. The protein was in $20 \mathrm{mM}$ Tris buffer at $\mathrm{pH} 7.5$ with $100 \mathrm{mM} \mathrm{NaCl}$. The signals that have not completely disappeared in the black spectrum belong mainly to residues located on mobile regions (G10, D21, T22, N25, Q31, D39, Q41, Q49, D52, R54, S57, D58, Y59, N60, L71, R72, L73, G76).
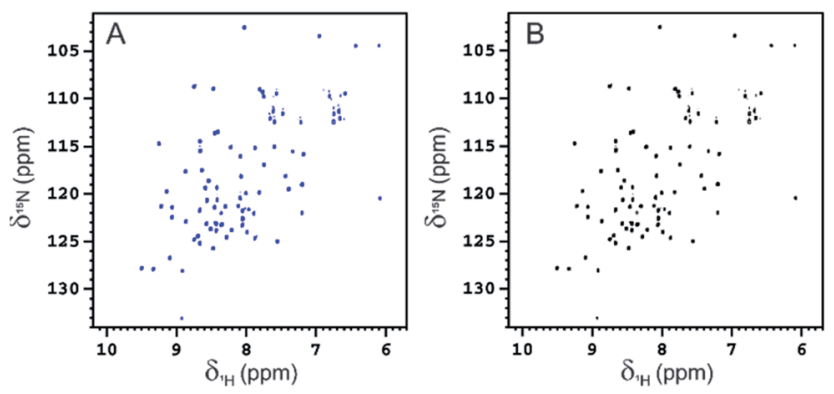

Fig. $102 \mathrm{D}{ }^{1} \mathrm{H}-{ }^{15} \mathrm{~N}$ HSQC spectra of free Rha-A28C (100 $\mu \mathrm{M}$, blue) and Rha-A28C in the presence of $50 \mu \mathrm{M}$ BambL (black).

we analyzed the viability of RAW 264.7 cells exposed to each test compound by MTT assay. No toxicity was observed after $24 \mathrm{~h}$ cell exposure to increasing concentrations $(0.01-1 \mu \mathrm{M})$ of each compound (Fig. S9†).

The ability of each compound to activate macrophages was, then, studied by analyzing the production of the proinflammatory cytokine TNF- $\alpha$ (Fig. 11). Both Fuc and Rha, when conjugated to Ub or Ub mutant (Fuc-Ub, Fuc-Rha-A28C, Rha-A28C), induced significant TNF- $\alpha$ production in a concentration-dependent manner. Conversely, neither Ub alone or un-conjugated saccharides (i.e. Fuc and Rha) stimulated TNF$\alpha$ release at any concentrations tested, suggesting that Fuc and Rha organization on the protein could be fundamental to induce macrophage activation. When the same compounds were added to LPS-stimulated RAW 264.7 cells the amount of TNF- $\alpha$ released resulted slightly decreased in comparison to cells treated with LPS alone. Of note, this reduction reached the statistical significance in samples treated with the orthogonally glycosylated Ub mutant, Fuc-Rha-A28C (Fig. 11).

Macrophages are part of the innate surveillance system deputed to kill bacteria by releasing soluble factors (i.e. nitrogen
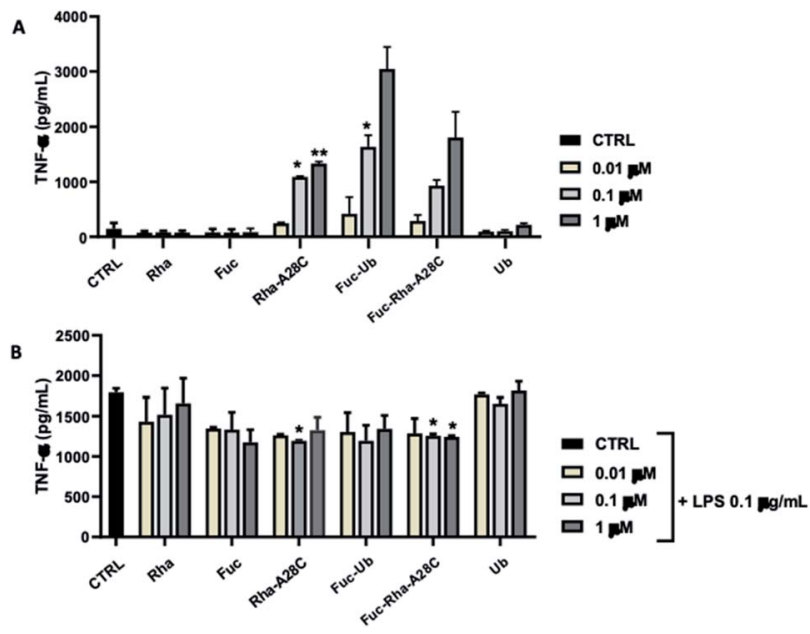

Fig. 11 Effects of the different compounds on production of the proinflammatory mediator tumor necrosis factor (TNF) - $\alpha$. RAW264.7 cells were seeded at a density of $0.6 \times 10^{5}$ cells per well in 24 -well plates, pre-treated with increasing concentrations $(0.01-1 \mu \mathrm{M})$ of each test compound for $1 \mathrm{~h}$ and then un-stimulated (A) or stimulated (B) with LPS $\left(0.1 \mu \mathrm{g} \mathrm{ml}^{-1}\right)$ for $24 \mathrm{~h}$. The data represent mean \pm SEM of at least three independent experiments run in triplicate.

and oxygenated reactive species), to recruit other immune cells by releasing cytokine to attenuate the infection, and to repair tissues after inflammatory stimuli. However, when macrophages are excessively activated (as during a severe infection in immune-depressed patients), a dysregulation between pro- and anti-inflammatory activities occurs and this leads to uncontrolled infections which tend to become chronic. The unprecedented effect observed for Fuc-Ub and in particular for Fuc-RhaA28C might open the way of the development of pathogen inhibitors with an immunomodulation activity.

\section{Conclusions}

Concluding, herein we proposed ubiquitin as scaffold protein and aryl- $\alpha$-O-fucoside as determinant to achieve conceptually new ligands with high affinity for Burkholderia ambifaria lectin, a pathogenic lectin responsible for severe infections in immunologically depressed or fibrosis cystic patients. To clarify the binding mode of aryl- $\alpha-O$-fucoside with lectin binding sites, the fucoside analogue 3 was prepared. Crystal structure of BambL complexed with 3 showed the fucoside moiety and aryl group well nested in all lectin sites. Moreover, as described for native fucose, an extensive interaction via hydrogen bonds connects the fucose-mimicking portion of $\mathbf{3}$ with the protein. Conversely, the flexible aliphatic portion of $\mathbf{3}$ is exposed to the solvent, poorly interacting with the protein. This arrangement and the high affinity assessed by ITC for $3 \mathrm{vs}$. BambL and AFL, prompted to synthesize derivative 7 to decorate Ub with multiple copies of aryl- $\alpha-O$-fucoside, which proved suitable exposed and oriented to bind with high affinity adjacent sites of the same BambL lectin. The interesting immunogenic properties of rhamnosecontaining polysaccharides recently isolated from medicinal plants, inspired the expression of Ub mutant A28C which 
displays a Cys residue in addition to the eight Lys residues. The resulting scaffold was orthogonally glycosylated by relying on two efficient click reactions to display one thio-rhamnose and up to eight residues of aryl- $\alpha$ - $O$-fucoside. Although rhamnose and fucose differ from the stereochemistry of only two stereocenters, rhamnose residue is not recognized by BambL binding sites nor interferes with lectin-aryl-fucoside binding. The three protein scaffolds Fuc-Ub, Rha-A28C and Fuc-Rha-A28C have been assayed for their immune modulator properties. The data obtained, even if preliminary, suggest that both Fuc and Rha efficiently presented to macrophages may be able to adjust physiological pathways of cell responses.

\section{Conflicts of interest}

There are no conflicts to declare.

\section{Acknowledgements}

SK was supported by the ANR/DFG French-German GLYCOMIME project (ANR-AAPG-2017). SK, EG and AI acknowledge support from Glyco@Alps (ANR-15-IDEX02), Labex Arcane/ CBH-EUR-GS (ANR-17-EURE-0003), Synchrotron SOLEIL, beamline PROXIMA 1 (Saint Aubin, France) and the help from Annabelle Varrot and beamline staff for data collection. SS was supported by MiUR (Prin 2015). MD was supported by European Union's framework program for Research and Innovation Horizon 2020 (2014-2020) under the Marie-Slodowska Curie Grant agreement number 675555, AEGIS. This work was also supported by Regione Toscana (CERM-TT and BioEnable). Authors acknowledge the support of Recombinant Proteins JOYNLAB, MIUR Italy PRIN 2017A2KEPL, and Progetto finanziato dalla Cassa di Risparmio di Firenze 2017 "Sviluppo di strategie innovative per la caratterizzazione a dettaglio atomico di coniugati proteina-polimeri per uso terapeutico (Bouncy NMR)". CN and MF thank MiUR (Progetto Dipartimenti di Eccellenza 2018-2022 allocated to Dept. of Chemistry).

\section{Notes and references}

$\S$ For general protein bioconjugation approaches see ref. 33-36.

1 O. Seitz, Chembiochem, 2000, 1, 214-246.

2 A. S. Shaw and E. L. Filbert, Nat. Rev. Immunol., 2009, 9, 4756.

3 A. Marra, J. Dong, T. Ma, S. Giuntini, E. Crescenzo, L. Cerofolini, M. Martinucci, C. Luchinat, M. Fragai, C. Nativi and A. Dondoni, Chem. - Eur. J., 2018, 24, 1898118987.

$4 \mathrm{H} . \mathrm{Hu}$ and S.-C. Sun, Cell Res., 2016, 26, 457-483.

5 S. Vijay-Kumar, C. E. Bugg and W. J. Cook, J. Mol. Biol., 1987, 194, 531-544.

6 H. Lis and N. Sharon, Chem. Rev., 1998, 98, 637-674.

7 J. Meiers, E. Siebs, E. Zahorska and A. Titz, Curr. Opin. Chem. Biol., 2019, 53, 51-67.
8 E. Mitchell, C. Houles, D. Sudakevitz, M. Wimmerova, C. Gautier, S. Pérez, A. M. Wu, N. Gilboa-Garber and A. Imberty, Nat. Struct. Biol., 2002, 9, 918-921.

9 A. Audfray, J. Claudinon, S. Abounit, N. Ruvoën-Clouet, G. Larson, D. F. Smith, M. Wimmerová, J. Le Pendu, W. Römer, A. Varrot and A. Imberty, J. Biol. Chem., 2012, 287, 4335-4347.

10 T. Dingjan, A. Imberty, S. Pérez, E. Yuriev and P. A. Ramsland, Front. Pharmacol., 2017, 8, 393.

11 C. Ligeour, O. Vidal, L. Dupin, F. Casoni, E. Gillon, A. Meyer, S. Vidal, G. Vergoten, J.-M. Lacroix, E. Souteyrand, A. Imberty, J.-J. Vasseur, Y. Chevolot and F. Morvan, Org. Biomol. Chem., 2015, 13, 8433-8444.

12 D. Hauck, I. Joachim, B. Frommeyer, A. Varrot, B. Philipp, H. M. Möller, A. Imberty, T. E. Exner and A. Titz, ACS Chem. Biol., 2013, 8, 1775-1784.

13 B. Richichi, A. Imberty, E. Gillon, R. Bosco, I. Sutkeviciute, F. Fieschi and C. Nativi, Org. Biomol. Chem., 2013, 11, 4086-4094.

14 D. Goyard, V. Baldoneschi, A. Varrot, M. Fiore, A. Imberty, B. Richichi, O. Renaudet and C. Nativi, Bioconjugate Chem., 2018, 29, 83-88.

15 J. Houser, J. Komarek, G. Cioci, A. Varrot, A. Imberty and M. Wimmerova, Acta Crystallogr., Sect. D: Biol. Crystallogr., 2015, 71, 442-453.

16 A. M. Boukerb, A. Rousset, N. Galanos, J.-B. Méar, M. Thépaut, T. Grandjean, E. Gillon, S. Cecioni, C. Abderrahmen, K. Faure, D. Redelberger, E. Kipnis, R. Dessein, S. Havet, B. Darblade, S. E. Matthews, S. de Bentzmann, B. Guéry, B. Cournoyer, A. Imberty and S. Vidal, J. Med. Chem., 2014, 57, 10275-10289.

17 Y. M. Chabre, D. Giguère, B. Blanchard, J. Rodrigue,

S. Rocheleau, M. Neault, S. Rauthu, A. Papadopoulos, A. A. Arnold, A. Imberty and R. Roy, Chem. - Eur. J., 2011, 17, 6545-6562.

18 K. Buffet, I. Nierengarten, N. Galanos, E. Gillon, M. Holler, A. Imberty, S. E. Matthews, S. Vidal, S. P. Vincent and J.-F. Nierengarten, Chem. - Eur. J., 2016, 22, 2955-2963.

19 N. Galanos, E. Gillon, A. Imberty, S. E. Matthews and S. Vidal, Org. Biomol. Chem., 2016, 14, 3476-3481.

20 H. Zhang, B. Wang, Z. Ma, M. Wei, J. Liu, D. Li, H. Zhang, P. G. Wang and M. Chen, Bioconjugate Chem., 2016, 27, 1112-1118.

21 M. K. Hossain, A. Vartak, P. Karmakar, S. J. Sucheck and K. A. Wall, ACS Chem. Biol., 2018, 13, 2130-2142.

22 V. Lehot, Y. Brissonnet, C. Dussouy, S. Brument, A. Cabanettes, E. Gillon, D. Deniaud, A. Varrot, P. Le Pape and S. G. Gouin, Chem. - Eur. J., 2018, 24, 19243-19249.

23 J. L. Asensio, A. Ardá, F. J. Cañada and J. Jiménez-Barbero, Acc. Chem. Res., 2013, 46, 946-954.

24 D. P. Gamblin, E. M. Scanlan and B. G. Davis, Chem. Rev., 2009, 109, 131-163.

25 M. Fernández-González, O. Boutureira, G. J. L. Bernardes, J. M. Chalker, M. A. Young, J. C. Errey and B. G. Davis, Chem. Sci., 2010, 1, 709-715.

26 M. M. Joullié and K. M. Lassen, Arkivoc, 2010, 8, 189-250. 
27 A. Arcangeli, L. Toma, L. Contiero, O. Crociani, L. Legnani, C. Lunghi, E. Nesti, G. Moneti, B. Richichi and C. Nativi, Bioconjugate Chem., 2010, 21, 1432-1438.

28 Comprehensive Organic Name Reactions and Reagents, ed. Z. Wang, American Cancer Society, 2010, pp. 3123-3128.

29 A. Dondoni, Synlett, 2020, 31, 1361-1371.

30 A. Dondoni, A. Massi, P. Nanni and A. Roda, Chem. - Eur. J., 2009, 15, 11444-11449.

31 A. Gimeno, P. Valverde, A. Ardá and J. Jiménez-Barbero, Curr. Opin. Struct. Biol., 2020, 62, 22-30.

32 M. Manuelli, S. Fallarini, G. Lombardi, C. Sangregorio, C. Nativi and B. Richichi, Nanoscale, 2014, 6, 7643-7655.
33 E. Ravera, S. Ciambellotti, L. Cerofolini, T. Martelli, T. Kozyreva, C. Bernacchioni, S. Giuntini, M. Fragai, P. Turano and C. Luchinat, Angew. Chem., Int. Ed. Engl., 2016, 55, 2446-2449.

34 S. Giuntini, E. Balducci, L. Cerofolini, E. Ravera, M. Fragai, F. Berti and C. Luchinat, Angew. Chem., Int. Ed., 2017, 56, 14997-15001.

35 S. Giuntini, L. Cerofolini, E. Ravera, M. Fragai and C. Luchinat, Sci. Rep., 2017, 7, 17934.

36 L. Cerofolini, S. Giuntini, A. Carlon, E. Ravera, V. Calderone, M. Fragai, G. Parigi and C. Luchinat, Chem. - Eur. J., 2019, 25, 1984-1991. 